\title{
Energy-Efficient Coverage Quality Guaranteed in Wireless Sensors Network
}

\author{
Tao Yang ${ }^{1, *}$, Dejun $\mathrm{Mu}^{1}$ and Wei $\mathrm{Hu}^{2}$ \\ ${ }^{1}$ School of Automation, Northwestern Polytecnical University, No.127 YouYi Road Xian, Shaanxi 710072, China \\ ${ }^{2}$ Department of Computer Science and Engineering, University of California, 9500 Gilman Drive, San Diego, CA 92093-0404, USA
}

Received: 20 Dec. 2012, Revised: 25 Apr. 2013, Accepted: 29 Apr. 2013

Published online: 1 Sep. 2013

\begin{abstract}
Wireless sensor network is always deployed randomly in specific area for intrusion detection and environmental monitoring. Due to the hostile environment, the sensor nodes could not be replaced easily. The efficient energy consumption scheme is known to be more and more important. Meanwhile, saving the energy could not impact the coverage quality. Connecting proper sensors adaptively as a barrier for monitoring is a good solution for both maximizing network lifetime and providing predetermined coverage ratio. The simulation results demonstrate that the algorithms can effectively reduce the energy consumption of the wireless sensor network and also keep the coverage quality on a high level.
\end{abstract}

Keywords: wireless sensor network, barrier coverage, energy efficient, quality

\section{Introduction}

Wireless sensor network with specifically capability to collect information from environment is deployed for monitoring and detecting military environment, battlefield, deserts, mountainous and border areas. It is constituted of a group number of tiny, cost-effective, power-efficient sensor nodes which can communicate over a specific distance. Replacing the sensor nodes is known to be the biggest problem in the wireless sensor network due to the hostile environment. Further be noted that the battery life is limited, so it reveals the importance of a good scheme to dispatch sensors in deployed region. On the other hand, since the limited number of sensor nodes in the deployed region, the quality of covering the area as high as possible has turned out to be one more objective demand to fulfill. The objective of guaranteeing an area coverage quality always tends to active sensor nodes as much as possible. Whereas, maximizing network lifetime will always try to active different sensor barriers in a rolling time or depend on the lifetime of entire barrier. Therefore both issues meet simultaneous optimization seems to be unreachable. Due to limited battery capacity, the wireless sensor nodes should avoid waste energy consumption during their work to increase the network lifetime. They need to manage their energy designedly. Many feasibility plans has been mentioned in [2], [4-11]. Grouping sensor nodes and turning redundancy nodes to sleep are the common way of saving the energy consumption in wireless sensor networks. The main goal of sensor scheduling algorithms is to turn off redundant sensor nodes and make them active when necessary. While providing at least one barrier coverage is a basic principle that must be followed. Some related researches proposed several solutions for maximizing the lifetime of wireless sensor network, while some papers presented the function for maximizing the coverage. They are asynchronous. So the equilibrium of both efficient energy and coverage quality is very important which needs to be studied. Localized Barrier Coverage Protocol (LBCP) is known to be the first localized sleep-wake up algorithm for movement detection applications has been presented in [13]. Sensing Neighborhood Cooperative Sleeping (SNCS), which performs dynamic scheduling among sensors depending on the amount of residual energy, has been proposed in [14]. In [15], researchers present an electrostatic field based coverage-enhancing algorithm (EFCEA) to examine the threshold value between coverage and network lifetime. Their results show that the coverage ratio can be enhanced much with

\footnotetext{
*Corresponding author e-mail: yangtao107@nwpu.edu.cn
} 
small number of the sensor nodes, whereas the network lifetime is prolonged with a large number of redundant sensor nodes. In the above papers, researchers focus on prolonging the network lifetime of WSN. Most of them present a scheme for utilizing residual energy of sensor nodes, while the measurement to become active or inactive at a specific time. In studies based on target-coverage, the status of neighbor nodes is also a crucial parameter need to be considered. Both coverage enhancement and network lifetime prolonging are essential to WSN. Sliding sensors algorithm in barrier (SSA) algorithms organize the working of sensor nodes and determine a set of active nodes to provide barrier coverage. A group of active nodes are replaced by sleeping nodes repeatedly. And problem of proper coverage ratio is well solved in the algorithm.

\section{Problem Statement: Coverage and Life Time in Wireless Sensor Network}

\subsection{Network model}

We firstly introduce some definitions.

Definition 1: Sensor Network, $N$ : A sensor network, $N$, is a collection of Sensors with the locations of sensor deployments.

Definition 2: Belt Region: A belt (region) has four boundaries. Two of them are parallel, and the other two are orthogonal to the two parallel ones. The Euclidean distance between two parallel boundaries is the width of the belt, $W$, while the Euclidean distance between two orthogonal boundaries is the length of the belt, $L$.

Definition 3: Coverage Graph, $G(N)$ : A coverage graph of a sensor network $N$ is constructed as follows. Let $G(N)$ $=(V ; E)$. The set $V$ consists of a vertex corresponding to each sensor. In addition, $V$ has two virtual nodes, $s$ and $t$ that correspond to the left and right boundaries, respectively. An edge exists between two nodes if their sensing regions overlap in the deployment region $R$. An edge exists between $u$ and $s$ (or $t$ ) if the sensing region of $u$ overlaps with the left boundary (or right boundary) of the region, Shown in Figure 1. Definition 4: Break Path:

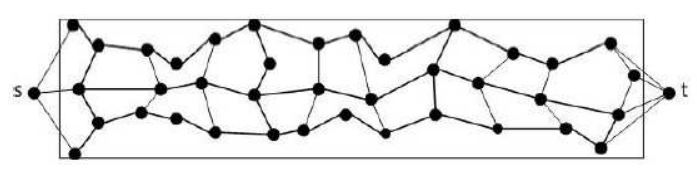

Fig. 1: The coverage graphic $\mathrm{G}(\mathrm{N})$

A break path is the trajectory of an intruder when he is in the belt. So the break path is assumed to connect the one side of the belt to the opposite. We assume that not all the break path are orthogonal, which is parallel to the belts left and right sides. So the shortest length of the break path is equal to the width $\mathrm{w}$ and some of them maybe longer.

Definition 5: Weak Zone, $Z_{i}$ : A weak zone is a slice of the belt region. Two of its edges coincide with the belts two parallel boundaries, and the other two edges are orthogonal break paths. We denote the longest line from left boundaries to right boundaries as the length of zone $\mathrm{Z}, L_{Z}$. The direction is orthogonal break path. The weak zone is always appear around the sink nodes.

Definition 6: $k$-barrier Coverage: A path is $\mathrm{k}$ covered if it intersects the sensing regions of at least $\mathrm{k}$ distinct sensors. A sensor network $\mathrm{N}$ provides $\mathrm{k}$-barrier coverage over a belt region $B$ if all paths through belt are k-covered by sensors in $N$.

We then introduce some notations as follow.

\begin{tabular}{cl}
\hline notations & meanings \\
\hline$R_{i}$ & Sensing range of sensor $i=1 \ldots n$ \\
$E_{r}$ & Ramaning energy \\
$t_{i}$ & The working time of sensor $r_{i} i=1 \ldots n$ \\
$A o_{i j}$ & Overlap area between sensor $_{i}$ and \\
& sensor $_{j} i=1 \ldots n, j=1 \ldots n, i j$ \\
$E_{i}$ & Initial energy reserve of sensor $_{i}$, \\
& $j=1 \ldots n, i j$ \\
$D_{i j}$ & Distance between sensor $i$ and $j$ \\
& $i, j=1 \ldots n$ \\
$e_{S}, e_{T}, e_{R}$ & Energy required for sensing, \\
& transmitting,receiving one unit data
\end{tabular}

We assume that a sensor has an initial energy capacity $e$, For two nodes $s_{i}$ and $s_{j}$, when $s_{i}$ transmitting one unit data to $s_{j}$, it costs energy $e^{T} d_{i j}^{\alpha}$, where $\alpha$ is the parameters of signal decline. Sensors obtain the distance from its neighbors by the GPS module in the disc.

\subsection{Coverage and lifetime}

The coverage and lifetime are competing with each other. This eventually implies the high energy consumption cause $e^{T} d_{i j}^{\alpha}$ and $e^{R} d_{i j}^{\alpha}$ is maximizing while the overlap is minimizing. On the other hand, in order to maximize lifetime, all the sensor nodes must reduce the energy consumption on transmitting and receiving. For a single sensor node, the energy consumption could be reduced if the distance between sensor nodes could be as close as possible, which means that the overlap is the bigger the better. But for entire network, it needs more sensor nodes, which may lead to more redundancy.

1) The upper bound of network lifetime: From the above notations, we could describe the network remaining energy as $E_{r}$.

$$
E_{r}=\sum_{i=1, j=1, i \neq j}^{n}\left\{E_{i}-\left[\left(e_{i}^{R}+e_{i}^{T}\right) d_{i j}^{\alpha}-e^{S}\right] \cdot t_{i}\right\}=0
$$


When the deployment is randomly, the network Lifetime nodes is related with $m$, which is the maximum number of node disjoint paths between $s$ and $t$. We assume the lifetime of an individual sensor node be unity. Then, the maximum lifetime for which the network $N$ can provide k-barrier coverage is at most $m / k$.

2) The critical condition for sliding barrier coverage: The coverage of network is represented in terms of senor range $R$ and overlap areas $A_{O}$.

$$
\text { MaximumCoverage }=\sum_{i=1, j=1, i \neq j}^{n}\left(\pi R_{\text {sensor }_{i}}^{2}-A o_{i j}\right)
$$

The coverage ratio is the percentage of a certain barrier coverage area in the whole belt.

$$
\text { Coverageratio }=\frac{\text { A Certain Barrier Coverage }}{\text { Maximum Coverage }}
$$

In the belt region, the whole cover area is $L \cdot w$. Once the network provides 1 barrier coverage, it needs to cover at least $L \cdot 2 R$ area. So the coverage ratio must be higher than $2 R / L$. This is a necessary condition for considering whether a set of sensor nodes could provide the 1 barrier coverage.

\section{Sliding Sensors in Barrier to Maximizing the Lifetime}

In sliding sensor network, the key point is to choose barrier in a rolling time. The sliding network could work and alternate all the time, which is different from some mobile sensors added in a fixed network. In the localized condition, the sliding barrier can change the break path so that the intruder could not follow a permanent path to penetrate a belt. At the same time, it also provides the localized algorithm for sensors to determine the sleep-wakeup scheme.

We assume the lifetime of the sensors is homogeneous initially. As we mentioned, if the distance between sensors are closer, the energy consumption will be lower. Hence, some barriers working time are longer than others because their lower load. After several times taking turn, the lifetime of sensor nodes become heterogeneous. In this section, we focus on maximizing lifetime in heterogeneous condition.

\subsection{Constructing and Sliding Barriers}

Constructing barriers in the belt region is the precondition of sliding sensors. In a belt region, there may be several different barrier lines. As shown in figure 2, here are at least two barrier lines in the region. We present an algorithm to find all disjoint barriers in a belt region.
$G(V S(s ; t), E)$ by making each sensor node a vertex in $V$ with a vertex capacity of the value of the nodesremaining energy, which is possible to measure the remaining lifetime, shown in Figure 2.

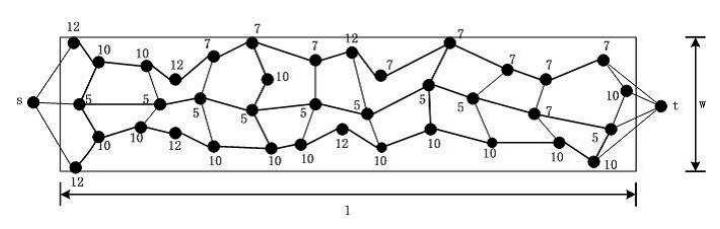

Fig. 2: The remaining energy graphic $G E(N)$

For any two vertices $u$ and $v$ in $V$, if their sensing areas overlap, then connect them. We add two nodes $s$ and $t$. For each node $u \in V$, if its sensing area intersects with the left boundary of the segment, connect $s$ to $u$; if its sensing area intersects with the right boundary of the segment, connect $u$ to $t$. We then use a standard algorithm, Edmond-Karp algorithm, to find a maximum flow from $s$ to $t$. Based on the above construction, nodes of the same path form a barrier that connects the left and right boundaries of the segment, and the maximum flow is the number of available disjoint barriers. And we also have to follow the equation mentioned before to satisfy the necessary condition of one barrier coverage.

\subsection{Sensors Sliding Algorithm}

We now formally establish the optimality of the Sensors Sliding Algorithm (SSA). The SSA algorithm determines a sleeping-wakeup scheme for single sensors. It provides $\mathrm{k}$-barrier coverage by the remaining network energy for the maximum network lifetime.

The Edmond-Karp algorithm computes the value of $f(N)$. If the nodes in the $G_{E}(N)$ are disjoint then the SSA are executed. We use this schedule to achieve the lifetime of $E_{r}(N)=k$. The complexity of the SSA is $O\left(k V^{3} / \log (V)\right)$.

\subsection{Mode Switching}

There are there modes for each sensor node in the network, active, sleeping or waking-up. Every nodes executes procedure initial to perform some preliminary work such as initializing variables(location, distance between neighbor sensors, belongs to which barrier line, etc). Then every active node periodically determine if it should stays active or go to sleep. A node may decide to go to sleep due to node redundancy or default rolling time. Meanwhile, it is important for every nodes to check whether falling asleep is jeopardizing the barrier property in the covered area. 


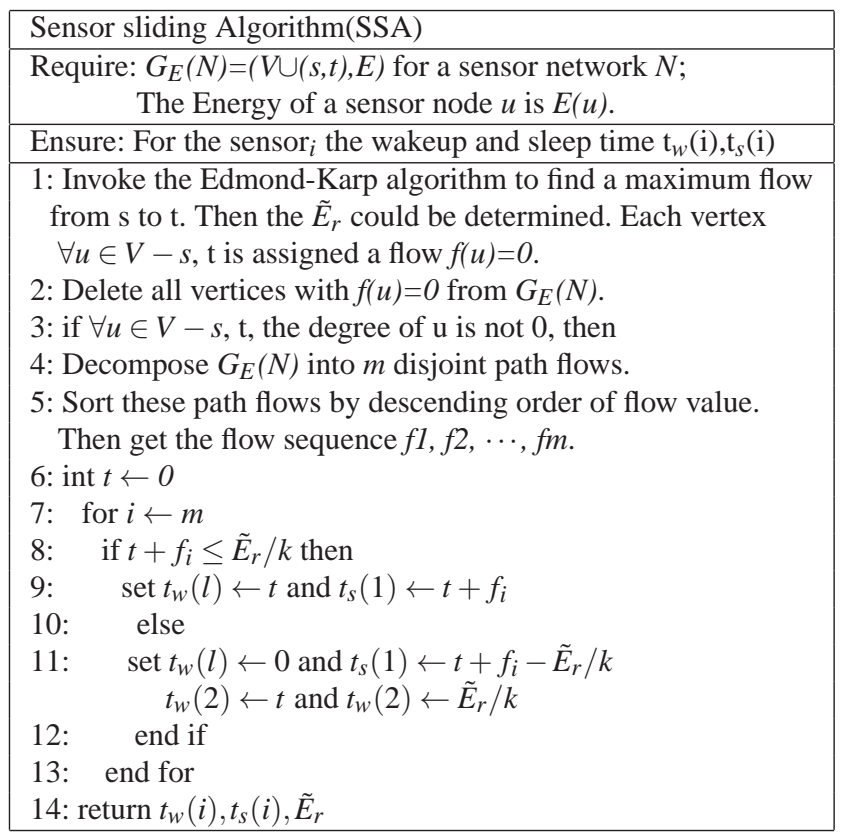

\section{Coverage Quality}

Once the coverage quality condition could be quantified, we then have the method to guarantee the coverage quality in the belt region.

\subsection{Definition of the coverage quality}

Definition 4.1 The Coverage Quality $Q_{k}$ : The coverage quality of a deployed belt region, denoted by $Q_{k}$, is related with the covered ratio in horizontal direction. The maximum $Q_{k}$ means the horizontal direction in belt region is fully covered while the minimum $Q_{k}$ means an orthogonal break path exists in the belt region. Definition 4.2 Overlap Sensing Boundary Zone, $Z n_{i}$ : Let $B\left(l_{i}, l_{i+1}\right)$ be a pair of one single sensors left and right sensing orthogonal boundaries. Let $\left(l_{0}, l_{1} l_{n}\right)$ be all the lines in $\mathrm{B}$ as ordered from left to right. We define $Z n\left(l_{i}, l_{i+n+1}\right)$ as a overlap sensing boundary zone $(1 \leq i \leq n)$.

\subsection{Guaranteeing the coverage quality}

We define two status of the coverage quality. $0<Q_{k}<1$ and $-1<Q k<0$. When $Q_{k}$ is between 0 and 1 , there are some redundancy sensors are deployed in the belt. In this situation, the SSA algorithm could be operated to prolong the lifetime of the whole network. When $Q_{k}$ is between - 1 and 0 , All the orthogonal crossing line in the belt region are k-barrier covered, but there are still some curvilinear break paths in the belt region, for example in Figure 3. Overlap sensing boundary zones could cover the belt region in horizontal direction but there are still interstices among the sensors. we should do some research on guaranteeing the coverage quality to prevent the risk of an intruder crossing the belt region by a fixed break path. We should notice that the smaller value of $Q_{k}$ in the situation, the more break paths exist in the belt.

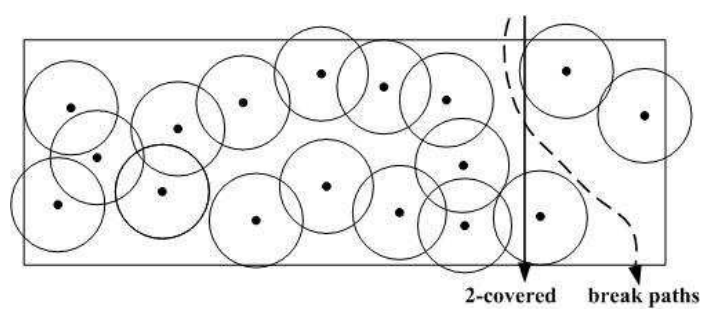

Fig. 3: Covered Condition if $-1<Q k<0$

If all the sensors are active in the belt region, the deployment could provide at least 1-covered and most areas are 2-covered. Furthermore, we could turn off some sensors to prolong the network lifetime and also satisfy the $-1<Q_{k}$ condition.

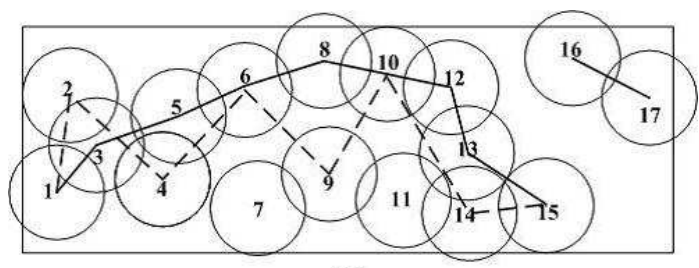

(a)

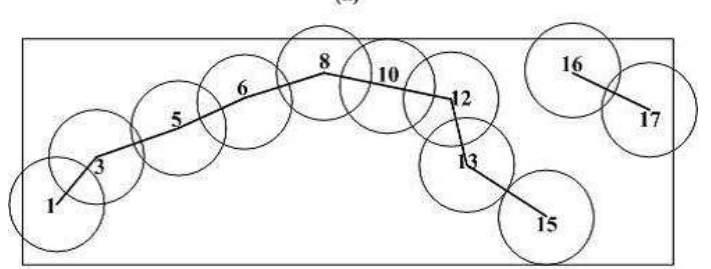

(b)

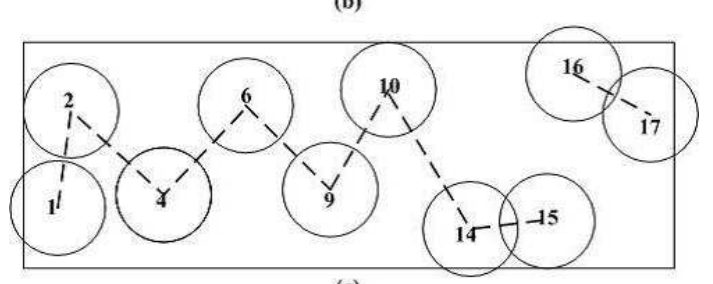

(c)

Fig. 4: improved method for constructing the barrier

There are two extreme cases in this deployed situation. As shown in Figure 4 (b) and (c). Obviously, the sensor selecting in (b) is better than it in (c). We denote them as line 1 and line2. So the scheme should consider both the barrier construction and the work time of different line. In the figure 4(b), the number of overlap sensor which is 9 , is 
much more than it in the figure 4(c). The work time of the barrier could be calculated according to the percentage of overlap sensors in the barrier. For example, the percentage of overlap sensors in figure 4(b) is $9 / 17$ and figure 4(c) is $2 / 17$. Furthermore, there are some sensors in a barrier that are not overlap with other ones, but they also cover some area to be a segment in the barrier. So the expression of a barrier work time is related with both the number of overlap sensors $\left(P B_{i}\right)$ in a barrier and the coverage ratio $\left(R B_{i}\right)$ of a barrier.

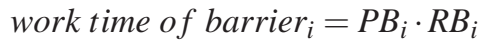

$$
\begin{aligned}
& P B_{i}=\frac{\text { the number of overlap sensor }}{j} \\
& R B_{i}=\frac{\text { a certain barrier coverage acreage }}{\text { maximum coverage acreage }}
\end{aligned}
$$

Based on the method mentioned above and the Sensors Sliding Algorithm (SSA), we present an improved algorithm to construct barriers and dispatch them. The Barrier Constructing Algorithm (BCA) considers both energy efficient and coverage quality.

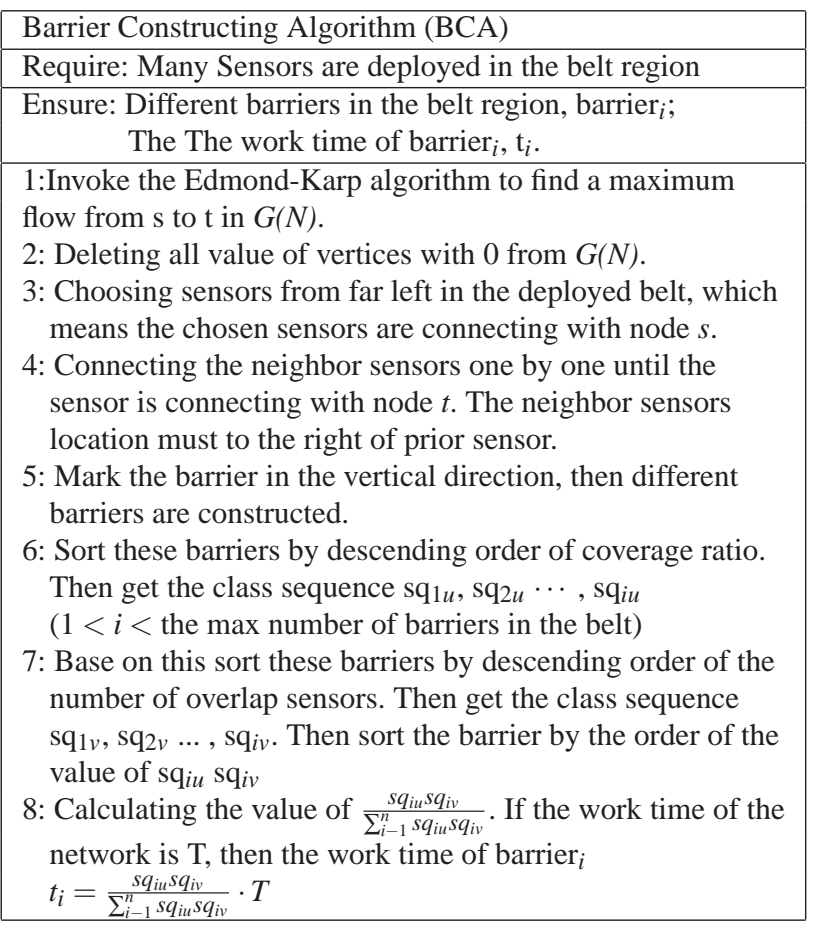

\section{Performance and Evaluation}

In the MATLAB simulation, sensors are deployed in the region of size $l \cdot w$ according to a two-dimensional uniform distribution. $l=1000 m$ and $w=200 m$, each sensor has a sensing range $R_{i}=25 \mathrm{~m}$. The $e_{S}=15 \mathrm{mAh}, e_{T}=$ $20 m A h, e_{R}=12 m A h$. We compare the result with the LBCP algorithm mentioned before. It is the first localized sleep-wakeup algorithm for movement detection applications that provided close to optimal enhancement in the network lifetime. Both the LBCP and the SSA aim to increase the lifetime of the network. Modeling the problem using an objective function which minimizes the energy consumption, instead of using an objective function which maximizes the network lifetime, it is possible to obtain better results. Shown in Figure 5 and Figure 6.

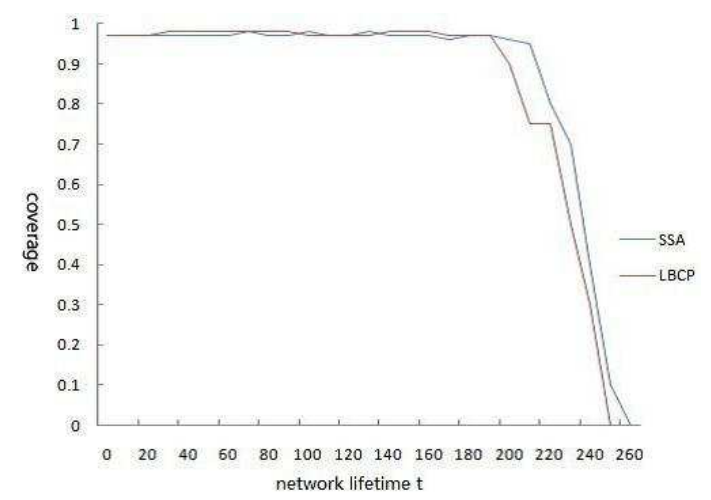

Fig. 5: The relationship between coverage and network lifetime

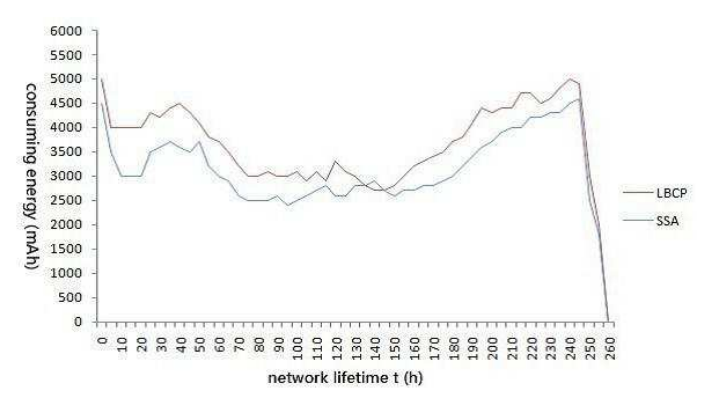

Fig. 6: Status of energy consumption

Since there is no algorithm identifying quality of coverage, we compare the performance of BCA with node replacement in general condition in barrier coverage of wireless sensor network. We have implemented algorithms BCA and the general replacement algorithm, which is mention in [15] (electrostatic field based coverage-enhancing algorithm) in MATLAB. The belt region is $2000 \mathrm{~m} \cdot 100 \mathrm{~m}$. Sensors were randomly deployed and the distribution and the initial energy of them are the same. The sensing range $R_{i}=25 \mathrm{~m}$. Ensureing $Q_{k}>0$, the 
number of replacement sensors are shown in Figure 7. The result shows that the BCA could replace less failed sensors. Meanwhile, the combination of SSA and BCA could both achieve required coverage quality and extend the lifetime of the whole network.

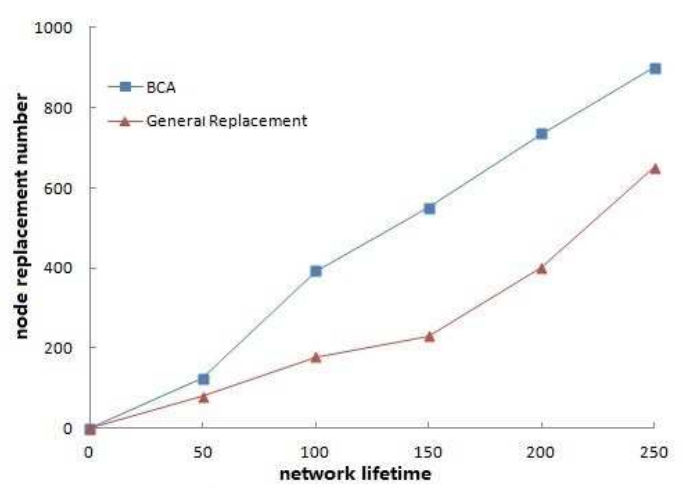

Fig. 7: The number of replacement node

\section{Conclusion}

Due to the small size of existing batteries sensors are suffering from their limited battery capacity. We present the algorithm to construct several barriers and formulate the sliding schemes for prolonging the entire network lifetime with a desired coverage quality. Reducing the energy consumption could not jeopardize desired coverage ratio for environment surveillance. Formulating a proper scheme to rolling the active work time, which is related to energy consuming activities, is the main focus of the research. Comparing with LBCP, the SSA formulates the scheduling mechanism by remaining energy and energy consumption. The different statuses of coverage quality are defined in the paper. Once sensors are deployed, the SSA and BCA algorithms could prevent the coverage quality from being short of the desired value and maximizing the lifetime of the wireless sensor network simultaneously.

\section{Acknowledgement}

This work is supported by the Aeronautical Science Foundation of China (2011ZD53046), (2013ZC53034) and the Aerospace Support Fund of China (2012-HT-XGD).

\section{References}

[1] M. Amac Guvensan, A. Gokhan Yavuz, On coverage issues in directional sensor networks: A survey, Ad Hoc Networks, 9, 1238-1255 (2011).
[2] H. Liu, P. Wan, C. W. Yi, X. Jia, S. Makki, N. Pissinou, Maximal lifetime scheduling in sensor surveillance networks, 4, 2482-2491 (2005)

[3] S. Kumar, T. H. Lai, and A. Arora, Barrier Coverage with Wireless Sensors, In Proc. of ACM MobiCom, (2005).

[4] S. Kumar, T. H. Lai, M. E. Posner, and P. Sinha. Optimal Sleep Wakeup Algorithms for Barriers of Wireless Sensors, In Fourth International Conference on Broadband Communications, Networks, and Systems IEEE BROADNETS, Raleigh, NC, (2007).

[5] Y. Yang, M. Cardei, Movement-assisted sensor redeployment scheme for network lifetime increase, in: Proc. of ACM Intl. Symposium on Modeling, Analysis, and Simulation of Wireless and Mobile Systems (MSWiM07), 13-20 (2007).

[6] J. Wang, C. Niu, R. Shen, A utility-based maximum lifetime algorithm for directional sensor network, in: Proc. of IEEE Intl. Conf. on Wireless Communications, Networking and Mobile Computing. WiCom07, Shanghai, China, 2424-2427, (2007).

[7] Study of key pre-distribution schemes in wireless sensor networks: case of BROSK (use of WSNet), Applied Mathematics \& Information Sciences, 5, 655-667 (2011).

[8] A. Bari, A. Jaekel, S. Bandyopadhyay, Clustering Strategies for Improving the Lifetime of Two-tiered Sensor Networks, Computer Communications, 31, 3451-3459 (2008).

[9] H. Liu, X. Jia, P. Wan, C.-W. Yi, S. Makki, and N. Pissinou, Maximizing Lifetime of Sensor Surveillance Systems, IEEE/ACM Transactions on Networking, 15, 334345 (2007).

[10] Y. Wu, S. Fahmy, N.B. Shroff, On the Construction of a Maximum-Lifetime Data Gathering Tree in Sensor Networks: NP Completeness and Approximation Algorithm, INFOCOM. (2008).

[11] Multi-Sensor Wireless Signal Aggregation for Environmental Monitoring System via Multi-bit Data Fusion, Applied Mathematics \& Information Sciences, 5, 589-603 (2011).

[12] E. J. DUARTE-MELO, M.LIU, Analysis of energy consumption and lifetime of heterogeneous wireless sensor networks. In Proceedings of the IEEE Global Telecommunications Conference. GLOBECOM. (2002).

[13] A. Chen, S. Kumar, and T.H. Lai, Designing localized algorithms for barrier coverage. In Proc. of ACM Mobicom, (2007).

[14] J. Ai, A. A. Abouzeid, Coverage by directional sensors in randomly deployed wireless sensor networks, Journal of Combinatorial Optimization, 11, 21-41 (2006).

[15] J. Zhao, J. C. Zeng, An electrostatic field-based coverageenhancing algorithm for wireless multimedia sensor networks, in: Proc. of IEEE Intl. Conf. on Wireless Communications, Networking and Mobile Computing (WiCom09), Beijing, China, 1-5 (2009). 


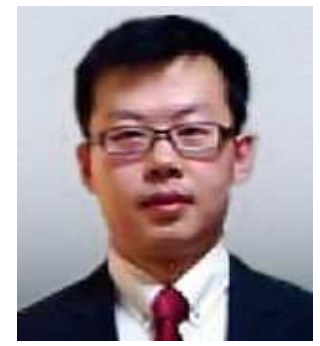

Tao Yang received the $\mathrm{PhD}$ degree in control theory and control engineering from Northwestern Polytechnical University, Xian, Shaanxi, China, in 2012. His research interests are in the areas of mathematical methods and models for complex systems, information security, the architecture of IOT. He has published research articles in reputed international journals of mathematical and engineering sciences.

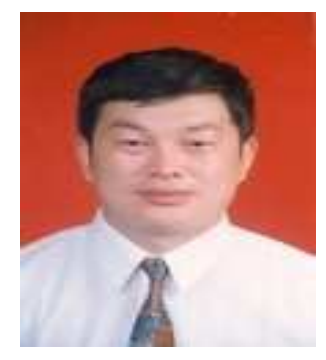

Dejun Mu received the Ph.D. degree in control theory and control engineering from Northwestern Polytechnical University, Xian, Shaanxi, China, in 1994. He is currently a Professor with the School of Automation, Northwestern Polytechnical University. His current research interests include control theories and information security, including basic theories and technologies in network information security, application specific chips for information security, and network control systems.

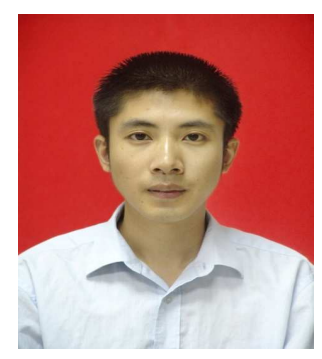

Wei $\mathbf{H u}$ is currently
pursuing the Ph.D.
degree from the School of Automation, Northwestern Polytechnical University, Xian, Shaanxi, China. $\mathrm{He}$ is a Visiting Graduate Student with the Department of Computer Science and Engineering, University of California, San Diego. His current research interests include security, reconfigurable devices, and embedded systems. 